\title{
Takayasu's Arteritis: An Uncommon Cause of Renal Artery Stenosis and Therapeutic Considerations
}

\author{
Sharad Virmani ${ }^{1}$, Luis Ortega ${ }^{2}$, Loay Salman ${ }^{1}$, Tushar Vachharajani ${ }^{3}$, Arif Asif ${ }^{4}$ and Ali Nayer ${ }^{*}, 1$ \\ ${ }^{I}$ Division of Nephrology and Hypertension, University of Miami, Miami, FL, USA \\ ${ }^{2}$ Division of Nephrology and Hypertension, Allegheny General Hospital, WPAHS, Pittsburg, PA, USA \\ ${ }^{3}$ Division of Nephrology and Hypertension W.G. (Bill) Hefner Veterans Affairs Medical Center, Salisbury, NC, USA \\ ${ }^{4}$ Division of Nephrology and Hypertension, Albany Medical College, Albany, NY, USA
}

\begin{abstract}
Takayasu's arteritis is a rare disorder characterized by granulomatous and necro-inflammatory disease of the aorta and its major branches. Its etiology remains unknown. We report a young woman with Takayasu's arteritis affecting the aortic arch, carotid, mesenteric, celiac and bilateral renal arteries resulting in severe hypertension, unilateral renal atrophy and renal insufficiency. The immunosuppressive therapy did not halt the progression of her vascular disease, which required revascularization procedures on numerous occasions. Here, the clinical manifestations and histopathological features of Takayasu's arteritis are reviewed. In addition, the available medical treatment options including glucocorticoids, cytotoxic agents and TNF-alpha inhibitors are discussed. Furthermore, current revascularization procedures such as percutaneous transluminal angioplasty and reconstructive vascular surgery in the treatment of occlusive vasculopathy due to Takayasu's arteritis are discussed. Although the prognosis of this debilitating disease has improved over the past two decades, a better understanding of its etiology and pathogenesis will facilitate the discovery of effective target-specific treatment strategies with a narrow adverse effects profile.
\end{abstract}

Keywords: Anti-TNFa, immunosuppression, percutaneous transluminal angioplasty, reconstructive surgery, renal artery stenosis, revascularization, Takayasu's arteritis, vasculitis.

\section{INTRODUCTION}

Takayasu's arteritis, also known as pulseless disease, aortic arch syndrome and non-specific aortoarteritis, is a rare granulomatous and necro-inflammatory disease of the aorta and its major branches. Here, a young woman with Takayasu's arteritis affecting the aortic arch, carotid, mesenteric and celiac arteries is presented. She developed bilateral renal artery stenosis resulting in severe hypertension, unilateral renal atrophy and renal insufficiency. The immunosuppressive therapy with methotrexate and prednisone did not halt the progression of the vascular disease. She underwent percutaneous transluminal angioplasty and reconstructive vascular surgery on numerous occasions. The clinical manifestations, pathogenesis and available treatment options for Takayasu's arteritis are discussed below. In addition, percutaneous and surgical treatment options for renal artery stenosis due to Takayasu's arteritis are presented.

\section{CASE REPORT}

At the time of current presentation, the patient was a 37year-old woman with a past medical history notable for Takayasu's arteritis and hypertension that was seen in the emergency department for progressive shortness of breath at

\footnotetext{
*Address correspondence to this author at the Clinical Research Building, Suite 825, 1120 NW 14th St., Miami, FL 33136, USA; Tel: 305.243.8491; Fax: 305.243.3506; E-mail: ANayer@med.miami.edu
}

rest for three weeks and elevated systolic blood pressures of $210 \mathrm{mmHg}$.

At age 23 years old, she presented with a syncopal episode. At that time an extensive work-up demonstrated left carotid artery stenosis, aortic valve regurgitation and aortic archstenosis. While no histological data is available from that hospitalization, she was diagnosed with Takayasu's arteritis based on clinical grounds and angiographic findings. She underwent extensive surgical and medical care. Surgical repair included a bypass graft from the ascending thoracic aorta proximal to the origin of the left carotid artery and aortic valve replacement with a bioprosthetic valve. With respect to medical care, she was started onmethotrexate and prednisone. Two years later, she experienced progressively worsening hypertension resistant to medical management and rising serum creatinine to $1.4 \mathrm{mg} / \mathrm{dl}$ from the baseline value of $0.6 \mathrm{mg} / \mathrm{dl}$. An aortogram revealed bilateral renal artery stenosis at the level of the ostia. Percutaneous transluminal angioplasty (PTA) and stentplacement was employed to the right and left renal arteries. The immunosuppressive therapy with methotrexateand prednisone was continued. Hypertension was treated with carvedilol, irbesartan and hydrochlorothiazide. Subsequently, the patient experiencedthree sequential episodes of hypertensive emergencies complicated by flash pulmonary edema and worsening azotemia. Angiography revealed critical in-stent restenosis of the right renal artery requiring PTA and re-stenting with bare-metal stents on two occasions 


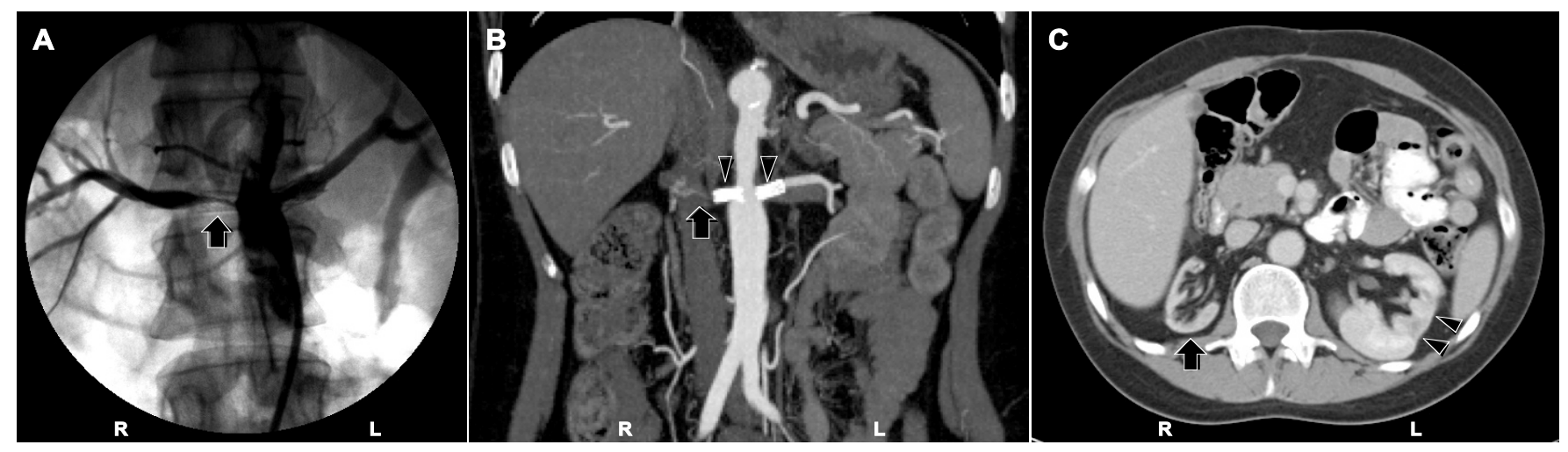

Fig. (1). Renal artery stenosis in Takayasu's arteritis. A. An intra-arterial (catheter) angiogram showing severe narrowing (arrow) of the right renal artery. B. Computed tomographic angiography showing stents (arrowheads) in both renal arteries and markedly attenuated blood flow to the right kidney (arrow). C. Abdominal computed tomography demonstrating atrophy of the right kidney (arrow) and compensatory hypertrophy of the left kidney. In addition, focal cortical thinning (arrowheads) of the left kidney is noted.

(Fig. 1A, B). Each exacerbation was considered secondary to inflammatory arterial stenosis from Takayasu's arteritis. Later, because of the abdominal pain a Doppler ultrasonography was performed which demonstrated poor blood flow to the superior mesenteric artery. A follow-up angiogram showed critical stenoses of the superior mesenteric artery and celiac artery. The patient then underwent bypass surgery that included a bifurcated bypass graft from the thoracic aorta with one limb to the common hepatic artery and a second limb to the superior mesenteric artery. Because of the recurrent right renal artery stenosis, a jump graft to the right renal artery distal to the stenosis was also performed during the surgical procedure. Following these procedures, the patient's blood pressure and renal function (serum creatinine: $1.4 \mathrm{mg} / \mathrm{dl}$ ) remained stable for 10 years.

At the time of current presentation, the patient was a young Hispanic woman in no acute distress. The temperature was $37.0{ }^{\circ} \mathrm{C}$, the blood pressure $210 / 110 \mathrm{~mm} \mathrm{Hg}$, the pulse 80 beats per minute, the respiratory rate 12 breaths per minute and the oxygen saturation $98 \%$ while breathing ambient air. Minimal crackles were auscultated at the lower lung fields bilaterally. A carotid bruit was present on the left, a 2/6 systolic blowing murmur along the right sternal border, a bruit present left of the umbilicus and a diminished left radial pulse. Serum creatinine and urea nitrogen were 1.7 $\mathrm{mg} / \mathrm{dl}$ and $55 \mathrm{mg} / \mathrm{dl}$, respectively. Cardiac catheterization revealed normal cardiac filling pressures with a normal functioning bioprosthetic aortic valve. Ultrasonography and abdominal computed tomography identified calcific plaques along the abdominal aorta, an atrophic right kidney measuring $6.5 \mathrm{~cm}$ in length and a left kidney measuring 11.8 $\mathrm{cm}$ in length (Fig. 1C). Doppler ultrasonography revealed elevated resistive indices in the left renal artery suggesting an in-stent stenosis. A left renal angiogram demonstrated $70 \%$ in-stent stenosis with a gradient of $63 \mathrm{mmHg}$ across the stenosis. The placement of a bare-metal stent in the left renal artery resulted in improved blood flow to the kidney. Following the intervention, serum creatinine declined to the baseline value of $1.4 \mathrm{mg} / \mathrm{dl}$.

\section{DISCUSSION}

First described by the ophthalmologist Mikito Takayasu in 1908, Takayasu's arteritis (TA) is a rare disorder characterized by a granulomatous and necro-inflammatory disease of aorta and its major branches [1]. TA affects predominantly women of childbearing age [2, 3]. The incidence of TA is estimated to be 2.6 and 150 per million per year in the United States and Japan, respectively $[2,3]$. The study of vascular inflammation in TA is hampered mainly due to the lack of accessibility to the inflamed arterial wall. Therefore, the pathogenesis of TA remains incompletely understood. Nonetheless, there is considerable overlap between TA and giant cell (temporal) arteritis; a related disorder that has been studied more extensively [4, 5].

\section{Clinical Features}

The clinical features of TA consist of constitutional symptoms (e.g. fatigue, headache, fever, unexplained weight loss, arthralgia, myalgia) and those related toischemia of the vascular territorydistal to the inflamed artery $[6,7]$. Constitutional symptoms may be lacking at presentation in $30 \%-50 \%$ of patients [6, 7]. Peripheral pulses are asymmetric or absent in $57 \%$ of patient at the time of first presentation [6]. The laboratory studies frequently reveal anemia, accelerated erythrocyte sedimentation, elevated serum level of C-reactive protein and hypergammaglobulinemia [2, 4]. The American College of Rheumatology proposes the following classification criteria for TA: onset at age $\leq 40$ years, claudication of an extremity, decreased brachial artery pulse, $>10 \mathrm{mmHg}$ difference in systolic blood pressure between arms, a bruit over the subclavian arteries or the aorta, and angiographic evidence of narrowing or occlusion of the entire aorta, its primary branches, or large arteries in the proximal upper or lower extremities [8]. When 3 or more of these 6 criteria are present, a diagnosis of TA can be rendered with a sensitivity of $90.5 \%$ and a specificity of $97.8 \%$. Renal arteries are affected in $24 \%$ to $76 \%$ of cases $[2,9,10]$. Renal artery stenosis and occlusion are the predominant findings. The involvement of renal arteries was bilateral in $79 \%$ of cases in which an angiographical examination of renal arteries was conducted [2]. However, the high prevalence of bilateral renal artery stenosis observed in the study by Hall and colleagues likely represents a selection bias. Hypertension is present in $33 \%$ to $70 \%$ of patient with TA and isoften a manifestation of renal artery stenosis $[2,6,10,11]$. Although plasma renin activity was 
elevated in patients with TA [12], there was no correlation between elevated plasma renin activity and renal artery stenosis [12]. Renal disease in TA is usually due to hypertension and/or ischemia as a result of renal artery stenosis.

\section{Pathology}

Upon gross examination, affected segments of arteries demonstrate thickening of the wall and adhesions to adjacent structures [9]. The luminal aspect of the segments involved often shows wrinkling (so-called tree barking). Regional lymph nodes draining inflamed arteries are often enlarged [9]. Pathological alterations of the vessel wall frequently result in luminal narrowing, aneurysmal dilatation, and/or thrombosis [9]. The histological examination demonstrates infiltration of the adventitia by mononuclear and polymorphonuclear inflammatory cells [9, 13]. At times, inflammatory cells in the adventitia aggregate to form distinct inflammatory nodules, reminiscent of lymphoid follicles. Immunohistochemical examination shows numerous lymphocytes (both B and T cells), dendritic cells, macrophages and neutrophils in the adventitia [13]. Immune reactions are especially conspicuous around vasa vasorum in the adventitia and at the interface between the adventitia and the media $[9,13]$. During the acute phase of inflammation, necrotic areas surrounded by multi-nucleated giant cells and mononuclear inflammatory cells may be found in the media. Over time, fibrous connective tissue and cells expressing alpha-smooth muscle actin replace the muscle layer of affected arteries [13]. Newly formed delicate blood vessels admixed with immune cells, mostly $\mathrm{T}$ lymphocytes and dendritic cells, may be found in the media and in the deep layer of the intima [13]. The intima reveals various degrees of fibrous thickening containing cells expressing alphasmooth muscle actin $[9,13]$.

\section{Management}

Over the past two decades, our understanding of the natural history of TA has improved, likely in part as a result of widespread non-invasive vascular imaging techniques [14]. In addition, the prognosis of TA has improved with the available treatment modalities [14]. Currently, available treatment options for TA consist of glucocorticoids, cytotoxic agents, inhibitors of tumor necrosis factor-alpha, percutaneous transluminal angioplasty and reconstructive vascular surgery. It is important to note that to date there have been no randomized controlled trials comparing efficacy of available treatment options and that each therapeutic intervention carry its own inherent risks and benefits.

Glucocorticoids. Glucocorticoids remain the first-line therapy for TA with the goal of disrupting the principal pathways of inflammation that result in arterial disease. Of note, the use of high-dose glucocorticoids has increased during the past decade [14]. A general consensus recommends an initial dose of prednisolone of 0.5-1.0 $\mathrm{mg} / \mathrm{kg} /$ day (maximum $60 \mathrm{mg} / \mathrm{day}$ ) for a month after which gradual tapering and maintenance doses of $10-15 \mathrm{mg} /$ day at 3 months can be used $[6,10,15]$. However, glucocorticoids can induce remission in only about $60 \%$ of patients $[10,16]$. In addition, glucocorticoid resistance and relapse during tapering of glucocorticoids are very common. When relapse was defined as new lesions in new vascular territories observed on vascular imaging, relapses occurred in more than $80 \%$ of patients studied at the National Institutes of Health and $90 \%$ of patients at the Cleveland Clinic $[6,10]$. The median dose of glucocorticoids at the time of relapse was $10 \mathrm{mg} /$ day. Thus, the addition of other immunosuppressive agents to glucocorticoids is recommended to achieve prolonged remission and to decrease toxicity associated with glucocorticoids [16].

Cytotoxic agents. Somewhere between $46 \%$ and $84 \%$ of patients with TA require additional immunosuppressive agents to induce and sustain remission [18]. Methotrexate (MTX, 15-25 mg/week) and azathioprine (AZA, $\leq 2$ $\mathrm{mg} / \mathrm{kg} /$ day) are the two cytotoxic agents that have been shown to induce and sustain clinical and angiographic remission when combined with glucocorticoids $[7,16,19$, 20]. In an open-label study, oral MTX and glucocorticoids induced remission in 13 of 16 patients with glucocorticoidresistant or relapsing TA [16]. Seven patients experienced relapses during glucocorticoid tapering. However, a remission could be successfully induced by MTX and glucocorticoid combination therapy in patients with a relapse. In addition to MTX, a combination therapy consisting of AZA and glucocorticoids administered to patients with active TA also resulted in attenuation of the systemic inflammatory response and stabilization of vascular lesions found on angiography [20]. However, no regression of the exsiting vascular lesions was observed. Based on theevidence that stems from comparative and case-control studies, the European League Against Rheumatism has statedthat the use of MTX and AZA as adjunct therapeutic agents to glucocorticoid therapy may ameliorate disease activity and diminish the cumulative glucocorticoid dose [15]. There is relatively little experience with cyclophosphamide in the treatment of TA [21]. Considering the toxicity profile of cyclophosphamide, its use is recommended in life-threatening TA that is refractory to other medical and surgical management.

Anti-TNF alpha antibodies. Produced mainly by activated macrophages and lymphocytes, tumor necrosis factor-alpha (TNF-alpha) is a proinflammatory cytokine that induces inflammation. TNF-alpha appears to play a role in the pathogenesis of TA [22]. Infliximab (a chimeric mouse and human monoclonal antibody) and etanercept (a soluble TNF receptor conjugated with the constant fragment of an IgG1 molecule) have been used as TNF-alpha inhibitors in the treatment of TA.

In a relatively recent multicenter open-label study, the treatment with infliximab and etanercept in patients with relapsing TA resulted in a complete or partial remission in 14 of 15 patients studied [17]. Before the treatment with TNF-alpha inhibitors, 13 of the 15 patients studied had been receiving glucocorticoids and additional immunosuppressive agents. Three patients developed adverse effects: one patient had an infusion reaction; another one developed disseminated histoplasmosis and a third patient experienced cutaneous herpes zoster infection.

In a separatestudy, infliximab was administered to 15 patients with refractory TA who had been treated with glucocorticoids $(n=14)$, MTX $(n=7)$ and AZA $(n=4)$ [23]. The addition of infliximab resulted in a significant clinical 
and biological response in $87 \%$ and $73 \%$ of patients at 3 and 12 months, respectively. On average, infliximab was administered at a dose of $5 \mathrm{mg} / \mathrm{kg}$ every 6 weeks. Five patients developed adverse effects including infusion-related reactions, pulmonary tuberculosis, severe cutaneous bacterial infection and Epstein-Barr virus reactivation. In a review of 84 published cases of TA refractory to the conventional immunosuppressive therapy that were treated with TNFalpha inhibitors, complete and partial remission was observed in $37 \%$ and $53 \%$, respectively [24]. After an average follow-up period of 10 months, significant adverse effects, mainly infections and hypersensitivity reactions, occurred in $20 \%$ of patients resulting in the discontinuation of TNF-alpha inhibitors in $10 \%$ of cases.

Based on the current literature, the inhibition of TNFalpha provides an alternative treatment strategy in TA that should be considered. Adverse effects to TNF-alpha inhibitors, however, are not uncommon. Because the treatment with TNF-alpha inhibitors can result in the activation of latent tuberculosis, patients who are considered for this therapy must have a negative skin test and a chest radiograph for tuberculosis.

Revascularization. Irreversible and symptomatic vascular lesions refractory to medical therapy have been treated with reconstructive vascular surgery and percutaneous interventions (PTA, stents). Such interventions are required in $20 \%$ to $44 \%$ of patients with TA depending on the artery involved and the severity of stenosis [10, 25]. Accepted indications for reconstructive vascular surgery in patients with TA include symptoms secondary to cervico-cranial vessel stenosis, coronary artery disease, severe aortic regurgitation or stenosis, hypertension with critical renal artery stenosis, limb claudication and progressive aneurismal enlargement [25]. Unless a vascular catastrophe is incipient, it is recommended to postpone reconstructive vascular surgery until clinical remission is achieved with medical intervention [7].

With regards to restenosis following revascularization, retrospective studies have compared surgical interventions to endovascular approaches. The Cleveland Clinic compared rates of restenosis in their cohort of 75 patients with TA after receiving either endovascular or surgical repair over a 14year period [6]. Although vascular patency was initially achieved in most patients, restenosis occurred more frequently in the PTA group (78\%)than in the surgical cohort (36\%) [6]. A more recent study explored outcomes in 25 patients with symptomatic occlusive disease involving the supra-aortic trunk (Subclavian, innominate, common and internal carotid arteries) following PTA or bypass surgery [26]. Among 15 arterial lesions treated with endovascular repair and 24 arterial lesions treated with a bypass, surgical treatment yielded a restenosis rate of only $12.5 \%$ while PTA resulted in $53.3 \%$. It must be recognized that these studies are observational by necessity and involve non-homogenous patient populations. Nevertheless, these studies examined relatively large numbers of patients and make an important contribution to literature.

Longevity of vascular patency following surgical reconstruction or PTA in patients with TA is of primary concern when deciding between the two modalities. While some studies endorse early success with PTA, reconstruction surgery provides lower long-term restenosis rates. However, other qualifying factors may play a role in predicting restenosis or complications following bypass or endovascular repair, such as length and severity of the arterial occlusion that make bypass more favorable to endovascular repair [25]. It is important to mention that lower restenosis rates are reported when either intervention is undertaken during the inactive stage of TA [7, 27-29]. However, many times revascularization procedures are unavoidable treatment strategies for patients at risk for organ failure from active disease. A serious long-term complication of vascular reconstruction surgery in patients with TA is anastomotic aneurysms [27, 28]. It occurred in $14 \%$ of patients followed over a 20-year period [27].

\section{Renal Artery Stenosis in TA}

TA-induced renal artery stenosis (TARAS) results in renal ischemia and hypertension $[2,9,10]$. The long-term effects of renal revascularization on renal function and blood pressure were examined in patients with TARAS who underwent aorto-renal bypass interventions [30]. The primary patency rates at 1,3 , and 5 years of follow-up were $87 \%, 79 \%$, and $79 \%$, respectively. Revascularization led to a significant decrease in blood pressure resulting in less antihypertensive agents to treat hypertension. On average, the estimated glomerular filtration rate increased by 12 $\mathrm{ml} / \mathrm{min}$. While no death was reported after the procedure, $19 \%$ of the patients developed significant postoperative complications including wound infection, myocardial infarction, mesenteric ischemia and retroperitoneal hematoma requiring repeat exploration. These findings are in line with those of a more recent study using autologous saphenous vein for bypass grafting in TARAS [31]. The primary patency rates at 1,3 , and 5 years of follow-up were $92 \%, 89 \%$, and $79 \%$, respectively. On average, the estimated GFR increased by $13 \mathrm{ml} / \mathrm{min}$ and systolic blood pressure decreased by $36 \mathrm{mmHg}$. There was no perioperative mortality. However, post-operative complications occurred in of $15 \%$ of patients and included retroperitoneal hematoma, transient ischemic attack and wound infection.

The results of PTA (with or without stent placement) in the treatment of TARAS have been less consistent. A retrospective study demonstrated that PTA for renal artery stenosis in patients with clinically inactive TA and hypertension resulted in a decrease in systolic blood pressure (by $45 \mathrm{mmHg}$ ) and the number of antihypertensive agents needed to control blood pressure [32]. As determined by worsening of hypertension and angiography, the rate of restenosis was $16 \%$ over the period of observation $(22 \pm 17$ months). In another retrospective study involving 12 patients with TARAS, PTA resulted in immediate patency in $83 \%$ of the patients. However, only $33 \%$ of the lesions were still patent at 5 years [33]. Another study showed that bypass/reconstruction surgery and PTA for TARAS resulted in $60 \%$ and $0 \%$ sustained patency, respectively, after a median follow-up period of 3 years [6].

As mentioned above, compared with PTA, surgical interventions result in a higher long-term patency for patients with TARAS. However, a perioperative complication rate of $15 \%-20 \%$ has been reported. Furthermore, anastomotic aneurysms represent an uncommon but serious long-term 
complication of vascular bypass surgery. Compared with surgical interventions, PTA isless invasive, less costly, technically less challenging, associated with less severe perioperative complications, and widely available. Considering the high prevalence of restenosis, a surveillance program for vascular patency following PTA is required. However, it remains to be determined what non-invasive imaging modality would provide the highest specificity and sensitivity in detecting TARAS. Computed tomographic (CT) and magnetic resonance (MR) angiography are not sensitive enough in the diagnosis of atherosclerotic renal artery stenosis due [34]. Compared with digital subtraction angiography (DSA), the sensitivity of CTA and MRA in detecting atherosclerotic renal artery sclerosis was $64 \%$ and $62 \%$, respectively [34]. Although DSA is considered the gold standard in the diagnosis of atherosclerotic renal artery sclerosis, it merely visualizes the arterial lumen [34]. Therefore, DSA often fails to detect vascular wall thickening during the earlier stages of TA when vascular patency is still unaffected.

\section{CONCLUSIONS}

The specific etiology and pathophysiology of TA remain largely obscure. Since its historic description to the present time, there have beenonly a few treatment strategies available for TA. While glucocorticoids remain the cornerstone of treatment, they fail to induce and sustain remission in a substantial proportion of patients. To provide further relief and reduce relapses, other immunosuppressive agents and TNF-alpha inhibitors are often required. In many cases, revascularization procedures become indispensible to reestablish organ perfusion. Bypass surgery appears to offer better long-term outcomes than percutaneous interventions. However, it is more costly, invasive, technically challenging and associated with more severe perioperative complications. In either case, a surveillance program for restenosis is needed. Controlled clinical trials are needed to compare the available treatment options for TA. However, considering the rarity of TA, such investigations are unlikely. It is hoped that large organization such as European League Against Rheumatism, the European Vasculitis Study Group and others can coordinate randomized controlled studies. Finally, a better understanding of the pathogenesis of TA will likely result in the development of novel and effective treatment strategies.

\section{CONFLICT OF INTEREST}

The authors confirm that this article content has no conflict of interest.

\section{ACKNOWLEDGEMENTS}

Declared none.

\section{REFERENCES}

[1] Takayasu M. A case with peculiar changes of the retinal central vessels (in Japanese). Acta Soc Ophthal Jpn 1908; 12: 554-5.

[2] Hall S, Barr W, Lie JT, Stanson AW, Kazmier FJ, Hunder GG. Takayasu arteritis. A study of 32 North American patients. Medicine 1985; 64: 89-99.

[3] Koide K. Takayasu arteritis in Japan. Heart Vessels Suppl 1992; 7: 48-54.

[4] Mason JC. Takayasu arteritis-advances in diagnosis and management. Nat Rev Rheumatol 2010; 6: 406-15.
[5] Weyand CM, Goronzy JJ. Medium- and large-vessel vasculitis. N Engl J Med 2003; 349: 160-9.

[6] Maksimowicz-McKinnon K, Clark TM, Hoffman GS. Limitations of therapy and a guarded prognosis in an American cohort of Takayasu arteritis patients. Arthritis Rheum 2007; 56:1000-9.

[7] Park MC, Lee SW, Park YB, Chung NS, Lee SK. Clinical characteristics and outcomes of Takayasu's arteritis: analysis of 108 patients using standardized criteria for diagnosis, activity assessment, and angiographic classification. Scand J Rheumatol 2005; 34: 284-92.

[8] Arend WP, Michel BA, Bloch DA, et al. The American College of Rheumatology 1990 criteria for the classification of Takayasu arteritis. Arthritis Rheum 1990; 33: 1129-34.

[9] Hotchi M. Pathological studies on Takayasu arteritis. Heart Vessels Suppl 1992; 7: 11-7.

[10] Kerr GS, Hallahan CW, Giordano J, et al. Takayasu arteritis. Ann Intern Med 1994; 120: 919-29.

[11] Lupi-Herrera E, Sánchez-Torres G, Marcushamer J, Mispireta J, Horwitz S, Vela JE. Takayasu's arteritis. Clinical study of 107 cases. Am Heart J 1977; 93: 94-103.

[12] Yoneda $S$, Nukada $T$, Imaizumi $M$, Miyai $M$, Abe $H$. Hemodynamic and volume characteristics, and peripheral plasma renin activity in Takayasu's arteritis. Jpn Circ J 1980; 44: 950-6.

[13] Inder SJ, Bobryshev YV, Cherian SM, et al. Immunophenotypic analysis of the aortic wall in Takayasu's arteritis: involvement of lymphocytes, dendritic cells and granulocytes in immunoinflammatory reactions. Cardiovasc Surg 2000; 8: 141-8.

[14] Ohigashi H, Haraguchi G, Konishi M, et al. Improved prognosis of Takayasu arteritis over the past decade - comprehensive analysis of 106 patients. Circ J 2012; 76: 1004-11.

[15] Mukhtyar C, Guillevin L, Cid MC, et al. European Vasculitis Study Group. EULAR recommendations for the management of large vessel vasculitis. Ann Rheum Dis 2009; 68: 318-23.

[16] Hoffman GS, Leavitt RY, Kerr GS, Rottem M, Sneller MC, Fauci AS. Treatment of glucocorticoid-resistant or relapsing Takayasu arteritis with methotrexate. Arthritis Rheum 1994; 37: 578-82.

[17] Hoffman GS, Merkel PA, Brasington RD, Lenschow DJ, Liang P. Anti-tumor necrosis factor therapy in patients with difficult to treat Takayasu arteritis. Arthritis Rheum 2004; 50: 2296-304.

[18] Kötter I, Henes JC, Wagner AD, Loock J, Gross WL. Does glucocorticosteroid-resistant large-vessel vasculitis (giant cell arteritis and Takayasu arteritis) exist and how can remission be achieved? A critical review of the literature. Clin Exp Rheumatol 2012; 30(1 Suppl 70): S114-29.

[19] de Franciscis S, Serra R, Luongo A, Sabino G, Puzziello A. The management of Takayasu's arteritis: personal experience. Ann Vasc Surg 2007; 21(6): 754-60.

[20] Valsakumar AK, Valappil UC, Jorapur V, Garg N, Nityanand S, Sinha N. Role of immunosuppressive therapy on clinical, immunological, and angiographic outcome in active Takayasu's arteritis. J Rheumatol 2003; 30: 1793-8.

[21] Shelhamer JH, Volkman DJ, Parrillo JE, Lawley TJ, Johnston MR, Fauci AS. Takayasu's arteritis and its therapy. Ann Intern Med 1985; 103: 121-6.

[22] Tripathy NK, Gupta PC, Nityanand S. High TNF-alpha and low IL2 producing $\mathrm{T}$ cells characterize active disease in Takayasu's arteritis. Clin Immunol 2006; 118: 154-8.

[23] Mekinian A, Néel A, Sibilia J, et al. Club Rhumatismes et Inflammation, French Vasculitis Study Group and Société Nationale Française de Médecine Interne. Efficacy and tolerance of infliximab in refractory Takayasu arteritis: French multicentre study. Rheumatology 2012; 51: 882-6.

[24] Comarmond C, Plaisier E, Dahan K, et al. Anti TNF- alpha in refractory Takayasu's arteritis: cases series and review of the literature. Autoimmun Rev 2012; 11: 678-84.

[25] Liang P, Tan-Ong M, Hoffman GS. Takayasu's arteritis: vascular interventions and outcomes. J Rheumatol 2004; 31:102-6.

[26] Kim YW, Kim DI, Park YJ, et al. Surgical bypass vs endovascular treatment for patients with supra-aortic arterial occlusive disease due to Takayasu arteritis. J Vasc Surg 2012; 55: 693-700.

[27] Miyata T, Sato O, Koyama H, Shigematsu H, Tada Y. Long-term survival after surgical treatment of patients with Takayasu's arteritis. Circulation 2003; 108: 1474-80.

[28] Takagi A, Tada Y, Sato O, Miyata T. Surgical treatment for Takayasu's arteritis. A long-term follow-up study. J Cardiovasc Surg 1989; 30: 553-8. 
[29] Saadoun D, Lambert M, Mirault T, et al. Retrospective analysis of surgery versus endovascular intervention in Takayasu arteritis: a multicenter experience. Circulation 2012; 125: 813-9.

[30] Weaver FA, Kumar SR, Yellin AE, et al. Renal revascularization in Takayasu arteritis-induced renal artery stenosis. J Vasc Surg 2004; 39: 749-57.

[31] Feng R, Wei X, Zhao Z, et al. Aortorenal bypass with autologous saphenous vein in Takayasu arteritis-induced renal artery stenosis. Eur J Vasc Endovasc Surg 2011; 42: 47-53.
[32]

Sharma S, Gupta H, Saxena A, et al. Results of renal angioplasty in nonspecific aortoarteritis (Takayasu disease). J Vasc Interv Radiol 1998; 9: 429-35.

[33] Fava MP, Foradori GB, García CB, et al. Percutaneous transluminal angioplasty in patients with Takayasu arteritis: fiveyear experience. J Vasc Interv Radiol 1993; 4: 649-52.

[34] Vasbinder GB, Nelemans PJ, Kessels AG, et al. Renal Artery Diagnostic Imaging Study in Hypertension (RADISH) Study Group. Accuracy of computed tomographic angiography and magnetic resonance angiography for diagnosing renal artery stenosis. Ann Intern Med 2004; 141: 674-82.

(c) Virmani et al.; Licensee Bentham Open.

This is an open access article licensed under the terms of the Creative Commons Attribution Non-Commercial License (http://creativecommons.org/licenses/by-nc/3.0/) which permits unrestricted, non-commercial use, distribution and reproduction in any medium, provided the work is properly cited. 